\title{
Comments on the article entitled "Morphologic changes after bladder neck intussusception in laparoscopic radical prostatectomy contribute to early postoperative continence"
}

\author{
Jieping $\mathrm{Hu}^{1}\left[\right.$ ? Qingke $\mathrm{Chen}^{1} \cdot \mathrm{Bin}_{\mathrm{Fu}}{ }^{1}$
}

Received: 20 February 2019 / Accepted: 26 February 2019 / Published online: 8 April 2019

C Springer Nature B.V. 2019

We recently read a paper entitled "Morphologic changes after bladder neck intussusception in laparoscopic radical prostatectomy contribute to early postoperative continence" in this journal [1]; the authors modified a bladder neck intussusception technique to restore early postoperative continence in laparoscopic radical prostatectomy. Several points should be addressed.

First, the author stated that the main aim of the present study was to explore the mechanism of "the intussusception technique" in improving postoperative early continence, but not to compare modified bladder neck intussusception with traditional intussusception. If the study want to emphasize the modified bladder neck intussusception technique, comparison should be made between modified bladder neck intussusception technique and non-modified bladder neck intussusception technique, not non-intussusception technique. Second, the cases for the research were relatively small (intussusception group, $n=10$ and non-intussusception group, $n=10$ ); although the baseline characteristics of the two groups appeared similar, randomized controlled trials with larger sample size are imperative in the future. Third, both of the intussusception group and non-intussusception group had one patient with positive surgical margin, it was hard to assess the effect of positive surgical margin on postoperative continence.

\section{Compliance with ethical standards}

Conflict of interest The authors declare that they have no conflict of interest.

\section{References}

1. Lin Y, Chen YK, Wang H et al (2019) Morphologic changes after bladder neck intussusception in laparoscopic radical prostatectomy contribute to early postoperative continence. Int Urol Nephrol. https://doi.org/10.1007/s11255-019-02118-0 (Accepted)

Publisher's Note Springer Nature remains neutral with regard to jurisdictional claims in published maps and institutional affiliations.
Jieping $\mathrm{Hu}$

hu_jieping@163.com

$\mathrm{Bin} \mathrm{Fu}$

792586870@qq.com

1 Department of Urology, The First Affiliated Hospital of Nanchang University, Nanchang 330,006, Jiangxi, China 